\title{
URBAN TANDEM-X RAW DEM FUSION BASED ON TV-L1 AND HUBER MODELS
}

\author{
H. Bagheri, M. Schmitt, X.X. Zhu \\ Signal Processing in Earth Observation, Technical University of Munich, Munich, Germany \\ Remote Sensing Technology Institute, German Aerospace Center, Oberpfaffenhofen, Wessling, Germany
}

\begin{abstract}
Recently, the TanDEM-X DEM has been produced as a global DEM with unprecedented relative accuracy. One important step of the chain of global DEM generation is to mosaic multiple raw DEM tiles by DEM fusion methods to reach the best possible target accuracy. Currently, Weighted Averaging (WA) is used as a fast and simple method for TanDEM-X raw DEM fusion in which the weights are computed from height error maps delivered from the Interferometric TanDEM-X Processor (ITP). In this paper, we investigate the efficiency of variational models such as TV-L1 and Huber model for the TanDEM-X raw DEM fusion task in comparison to WA. The results illustrate that using variational models can improve the quality of DEM fusion outputs especially for areas with high-frequency contents and more complex morphological features like urban areas. Using variational models could improve the DEM quality by up to about $1 \mathrm{~m}$.
\end{abstract}

Index Terms - Data fusion, $L_{1}$ norm total variation, Weight map, Huber model, TanDEM-X DEM

\section{INTRODUCTION}

A new global Digital Elevation Model (DEM) covering almost the whole planet was realized by the TanDEM-X DEM mission. The bistatic SAR data takes are input to a SAR interferometric processing chain to produce a DEM with relative height accuracy better than $2 \mathrm{~m}$ for slopes lower than $20 \%$ and finer than $4 \mathrm{~m}$ for remaining steeper slopes [1]. The main part of the DEM generation procedure is implemented in the Integrated TanDEM-X Processor (ITP) which leads to primary raw DEMs for each bistatic acquisition. After some pre-processing such as phase unwrapping, and DEM tile calibration with ground control points such as those derived from ICESat data, DEM scenes are fused together within the DEM Mosaicking Processor (DMP)[2].

A common method for doing the DEM fusion in DMP is weighted averaging in which the heights are summed respective to their weights derived from a Height Error Map (HEM). In addition, some logic for clustering consistent heights and upgrading weights regarding the influences of other significant factors such as Height of Ambiguity (HoA), Phase Unwrapping (PU) methodology and pixel locations relative to the border of the DEM scene is considered to finally reach the target relative accuracy and minimize PU errors remaining from primary steps [3]. While the weighted averaging approach can realize the predefined goals in DMP for global DEM generation it does not perform optimally in difficult terrains with complex morphology such as urban areas which contains many high-frequency contents such as edges. After WA-based DEM fusion, visualization shows that outlines of buildings are not perfectly sharp and still some amount of existing noise spoils the footprints of buildings. Therefore, we will investigate the application of more sophisticated approaches to efficiently preserve edges and outlines of buildings while removing noise. For this purpose, two variational models, namely $L_{1}$ norm total variation (TV- $L_{1}$ ) and Huber model are implemented.

\section{METHODS FOR TANDEM-X RAW DEM FUSION}

Different methods have been implemented for fusing DEMs with different properties [4], [5],[6] as an application of data fusion in remote sensing [7] . In this paper, three approaches are carried out for TanDEM-X raw DEM fusion. The description of each model will be explained in the following. Before implementing the fusion process, two DEMs are aligned to each other. This can easily be performed by DEM coregistration approaches such as least square matching, iterative closest point or manually decreasing of their translational and rotational discrepancies. For stability reasons, in addition, the height data should be normalized to [l $\left.\begin{array}{ll}0 & 1\end{array}\right]$ interval:

$$
h_{k}^{n}(x, y)=\frac{h_{k}(x, y)-h_{\min }}{h_{\max }-h_{\min }}
$$

where $h_{k}(x, y)>0$ is the elevation of the study DEM with index of $k$ in the location $(x, y), h_{\max }>0$ and $h_{\min }>0$ $\left(h_{\min }<h_{\max }\right)$ are lowest and highest elevations in whole study DEMs. The output gives the normalized height in the considered location.

\subsection{Weighted Averaging}

The most popular, very fast and low computational cost method for DEM fusion is weighted averaging which is implemented as follows [3]: 


$$
\mathbf{f}=\sum_{i=1}^{k} \mathbf{w}_{i} \odot \mathbf{h}_{i}
$$

where $\mathbf{h}_{i}$ are primary DEMs with normalized heights achieved from relation 1 and $\mathbf{w}_{i}$ are corresponding weight maps.

The main critical issue for using weighted averaging for DEM fusion is to apply appropriate weights that are fairly representative of expected height errors in the source DEMs. For TanDEM-X DEM fusion, generally, these weights are delivered as Height Error Maps (HEMs) from ITP. For each height of the TanDEM-X DEM, the corresponding HEM value can be estimated by

$$
\sigma_{j}=H_{a m b} \frac{\sigma_{\phi, j}}{2 \pi}
$$

where $H_{a m b}$ is the height of ambiguity and $\sigma_{\phi, j}$ is the interferometric phase error that is estimated from the interferometric coherence with geometrical consideration. Then, from these values, the respective weights can be calculated for each pixel location by:

$$
w_{j}=\frac{\frac{1}{\sigma_{j}^{2}}}{\sum_{j=1}^{N} \frac{1}{\sigma_{j}^{2}}}
$$

\subsection{Variational Models}

The basic variational model for image denoising and data fusion is a quadratic model in which $L_{2}$ norm is used for both regularization and data terms. However the quadratic regularization term causes over-smoothing for edges. Therefore, using the $L_{1}$ norm instead was proposed by Rudin, Osher, and Fatemi which is called ROF model correspondingly [8]. Since the ROF model still uses the $L_{2}$ norm for the data term, it does not provide robustness against outliers when applied to DEM fusion. As a solution, the $L_{1}$ norm can be substituted for $L_{2}$ norm. The TV- $L_{1}$ model consists of two terms, namely the data fidelity and the penalty term [9]:

$$
\min _{\mathbf{f}}\left\{\sum_{i=1}^{k}\left\|\mathbf{f}-\mathbf{h}_{i}\right\|_{1}+\gamma\|\nabla \mathbf{f}\|_{1}\right\}
$$

where $\mathbf{h}_{k}$ are noisy input DEMs and $\mathbf{f}$ is the desired DEM should be achieved by minimizing the functional energy above. The penalty term is formed based on the gradients of the newly estimated DEM to preserve the edges at the end. The regularization parameter $\gamma$, trades off between penalty and fidelity terms.

While the main advantage of TV- $L_{1}$ is its robustness against the strong outliers as well as preserving the edges, it suffers from the staircasing effect, a phenomenon that creates artificial discontinuities in the final output and particularly affects high resolution DEM fusion [10]. Moreover, the $L_{1}$ norm is not the necessarily the best choice for all data fusion and denoising cases. As an alternative, the Huber regularization model is proposed to rectify the drawbacks of TV- $L_{1}$ model. It applies the Huber norm instead of $L_{1}$ norm in both fidelity and penalty terms [11]:

$$
\|x\|_{\eta}= \begin{cases}\frac{|x|^{2}}{2 \eta} & \text { if }|x| \leq \eta \\ |x|-\frac{\eta}{2} & \text { if }|x| \geq \eta .\end{cases}
$$

where $\eta$ is a parameter that determines a threshold between the $L_{1}$ and $L_{2}$ norm in the model. Then, Huber model can be defined as follows:

$$
\min _{\mathbf{f}}\left\{\sum_{i=1}^{k} \sum_{\Omega}\left\|\mathbf{f}-\mathbf{h}_{i}\right\|_{\alpha}+\gamma \sum_{\Omega}\|\nabla \mathbf{f}\|_{\beta}\right\}
$$

where both data and penalty terms are constituted based on the thresholds $\alpha$ and $\beta$. Since, both TV- $L_{1}$ and the Huber model are convex, we use the primal-dual strategy to minimize the energy functional of variational models and consequently find the optimum fused DEM.

\section{EXPERIMENT AND RESULTS}

\subsection{Study areas}

As an experiment of using variational model for TanDEM$\mathrm{X}$ raw DEM fusion over urban areas, we selected study areas from two nominal existing tiles (I) and (II) and one nonofficial product (III) produced by ITP over Munich city in Germany. The specifications of all raw DEMs are shown in Tab. 1.

The main property that discriminates the three tiles is that the height of ambiguity is larger for tile (III) in comparison to (I) and (II), which means data takes of tiles (I) and (II) were acquired using a longer baseline. The DEM with the larger HoA will be helpful for areas where PU errors are dominant.

\begin{tabular}{lccc}
\hline \multicolumn{4}{c}{ TanDEM-X raws DEMs } \\
Tile index & $\mathrm{I}$ & $\mathrm{II}$ & $\mathrm{III}$ \\
\hline Center incidence angle & $38.25^{\circ}$ & $37.03^{\circ}$ & $38.33^{\circ}$ \\
Equator crossing direction & Ascending & Ascending & Ascending \\
Look direction & Right & Right & Right \\
Polarization & $\mathrm{HH}$ & $\mathrm{HH}$ & $\mathrm{HH}$ \\
Height of ambiguity & $45.81 \mathrm{~m}$ & $53.21 \mathrm{~m}$ & 72.02 \\
Total number of looks & 22 & 22 & 22 \\
Pixel spacing & $0.2 \mathrm{arcsec}$ & $0.2 \mathrm{arcsec}$ & $0.2 \mathrm{arcsec}$ \\
HEM mean & $1.33 \mathrm{~m}$ & 1.58 & 2.58 \\
\hline
\end{tabular}

Table 1. Properties of the TanDEM-X raw DEM tiles for Munich area.

After resampling and coregistration, the raw DEMs are fused by the different approaches explained in Section 2.

In this study two experiments will be carried to evaluate the performance of variational models in comparison to 


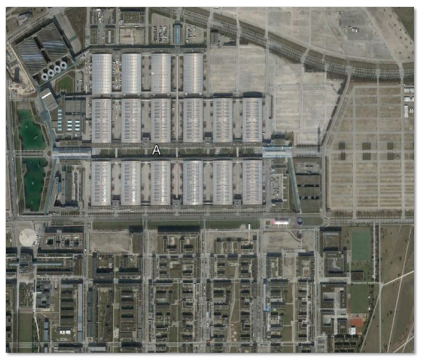

(a) Study area 1

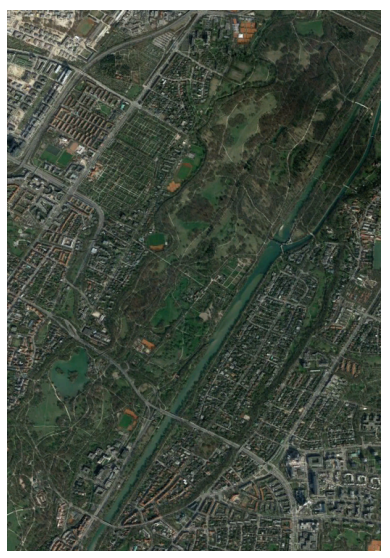

(b) Study area 2

Fig. 1. Display of study areas 1 and 2.

weighted averaging. In the first experiment, two patches from official tiles (I) and (II) are selected to investigate how the variational models perform in normal urban areas and in the second experiment, the patches are from tile (I) and (III) with considering an area has lots of inconsistent heights due to PU errors. Fig. 1 displays the study areas 1 and 2 selected in this study for the first and second experiments respectively.

\subsection{DEM Fusion Results}

Tab. 2 represents qualities of fused DEMs with different measures for the first study area (1). Fig. 2 displays the primary DEM patches and fusion results in a 3D manner. The accuracies have been measured with respect to a reference LiDAR DSM that is derived from a LiDAR point cloud with a density of one point per square meters after resampling into a grid with a pixel size of $6 \mathrm{~m}$. The results of the first investigation (area 1) illustrate using variational models can obtain a more accurate DEM in comparison to simple weighted averaging. The DEM fusion results of the second experiment are collected in Tab. 3. Unfortunately for this area, the reference data was not available and thus, the performance of the fusion algorithm is just evaluated based on the PU error reduction according to instructions described in [3].

\section{DISCUSSION}

The results of TanDEM-X DEM fusion confirm the better performance of variational models. Using TV- $L_{1}$ model for TanDEM-X raw DEM fusion could finally improve the quality of raw DEMs (I) and (II) up to 0.70 and $1 \mathrm{~m}$ (in term of RMSE value) respectively in the first study patch (area 1). In addition, revealing outlines of buildings in final fused DEM using weighted averaging is not as great as variational approaches that clarifies the superiority of variational models

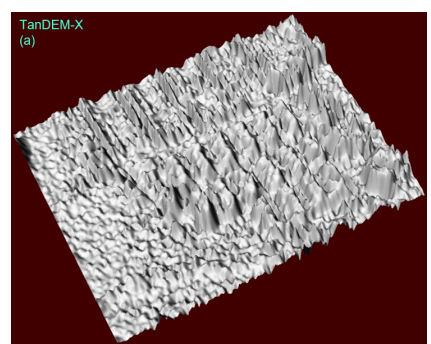

(a) TanDEM-X (tile I)

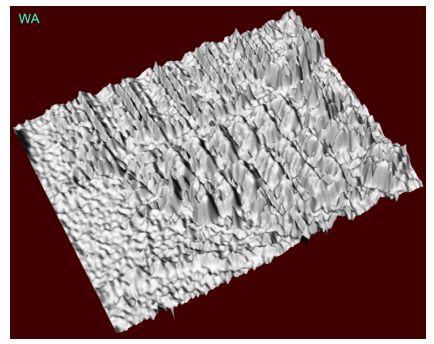

(c) Weighted averaging

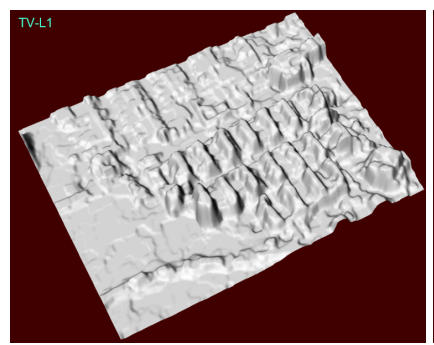

(e) TV-L1 model

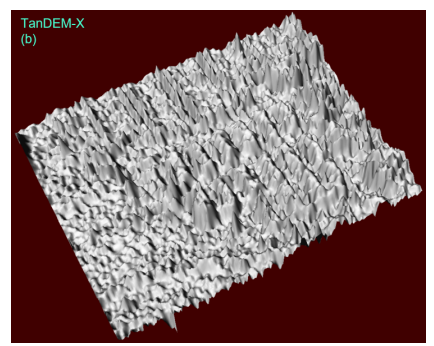

(b) TanDEM-X (tile II)

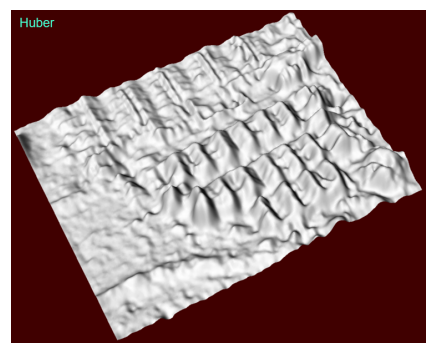

(d) Huber model

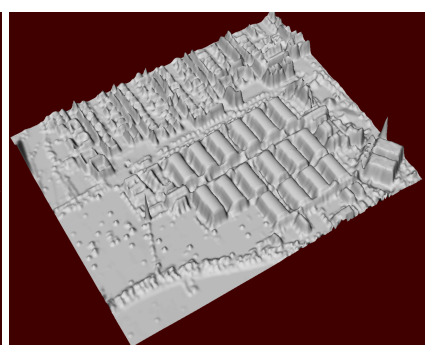

(f) LiDAR
Fig. 2. 3D display of initial TanDEM-X data and results of DEM fusions using different methods in the industrial area.

over weighted averaging in urban areas. The quantitative results identify there are no significant differences between the performance of TV- $L_{1}$ and the Huber model while the DEMs produced by $\mathrm{TV}-L_{1}$ have slightly better accuracy. However, an inspection of the 3D view of the Huber-DEM visualized in Fig. 2 implies that the Huber model tends to create a smoother output especially on the edges that are not very beneficial for DEMs with lower resolution. In the second investigation (area 2), it can be observed in areas influenced with PU errors choosing the optimal norm is essential to reach optimal results from DEM fusion. For this, two tiles, one with small HoA and and another with larger HoA should be employed to reduce the influence of phase unwrapping errors.

\section{CONCLUSION}

In this paper, we proposed to apply variational models (TV$L_{1}$ and Huber models) for TanDEM-X raw DEM fusion at the phase of DEM mosaicking instead of weighted averaging. The results illustrated that variational models are more 


\begin{tabular}{ll|ccccc}
\hline \multicolumn{1}{c|}{ DEM } & & Mean & RMSE & MAE & NMAD & STD \\
TanDEM-X (I) & & 0.711 & 4.395 & 3.082 & 2.373 & 4.338 \\
TanDEM-X (II) & & 0.711 & 4.638 & 3.273 & 3.005 & 4.583 \\
& WA & 0.766 & 4.157 & 2.932 & 2.244 & 4.086 \\
Fused DEM & TV- $L_{1}$ & 0.690 & 3.666 & 2.691 & 2.027 & 3.600 \\
& Huber & 0.711 & 3.742 & 2.842 & 2.400 & 3.674 \\
\hline
\end{tabular}

Table 2. Height accuracy (in meter) of the TanDEM-X data before and after DEM fusion in the study area 1.

\begin{tabular}{|c|c|c|c|c|}
\hline \multicolumn{2}{|c|}{ TanDEM-X DEM } & No. of PU errors & Max discrepancy & Min discrepancy \\
\hline \multicolumn{2}{|c|}{ Relative difference: (III-I) } & 762 & 79.201 & -46.671 \\
\hline \multirow{3}{*}{ Fused DEM } & WA & 73 & 51.771 & -30.832 \\
\hline & $\mathrm{TV}-L_{1}$ & 177 & 54.985 & -27.936 \\
\hline & Huber & 25 & 39.935 & -22.322 \\
\hline
\end{tabular}

Table 3. The performance of variational models and weighted averaging for reducing effects of PU errors in the problematic area (2).

efficient than weighted averaging for TanDEM-X raw DEM fusion especially over urban areas.

In the future, we will investigate on more datasets with different properties such as different HoAs as well as areas selected from different land types for instance residential areas, and inner city and also tiles produced from completely different baseline configuration, ascending and descending data takes in morphologically complex terrains and also fusion of TanDEM-X DEMs produced by non-local InSAR filtering.

\section{ACKNOWLEDGMENT}

The authors would like to thank Dr. Fritz of DLR and Mr. Baier for providing the TanDEM-X raw DEM; and the Bavarian Surveying Administration for providing the LiDAR data.

\section{REFERENCES}

[1] G. Krieger, A. Moreira, H. Fiedler, I. Hajnsek, M. Werner, M. Younis, and M. Zink, "TanDEM-X: A Satellite Formation for High-Resolution SAR Interferometry," IEEE Trans. on Geosci. and Remote Sens., vol. 45, no. 11, pp. 3317-3341, 2007.

[2] P. Rizzoli, M. Martone, C. Gonzalez, C. Wecklich, D. B. Tridon, B. Brutigam, M. Bachmann, D. Schulze, T. Fritz, M. Huber, B. Wessel, G. Krieger, M. Zink, and A. Moreira, "Generation and performance assessment of the global TanDEM-X digital elevation model," ISPRS Journal of Photogrammetry and Remote Sens., vol. 132, no. Supplement C, pp. 119 - 139, 2017.

[3] A. Gruber, B. Wessel, M. Martone, and A. Roth, "The TanDEM-X DEM mosaicking: Fusion of multiple acquisitions using InSAR quality parameters," IEEE Journal of Selected Topics in Applied Earth Observ. and Remote Sens., vol. 9, no. 3, pp. 1047-1057, March 2016.
[4] P. Reinartz, R. Müller, D. Hoja, M. Lehner, and M. Schroeder, "Comparison and fusion of DEM derived from SPOT-5 HRS and SRTM data and estimation of forest heights," in Proc. EARSeL Workshop on 3DRemote Sens., Porto, vol. 1, 2005.

[5] H. Papasaika, E. Kokiopoulou, E. Baltsavias, K. Schindler, and D. Kressner, "Fusion of digital elevation models using sparse representations," in Proceedings of the 2011 ISPRS Conference on Photogrammetric Image Analysis, ser. PIA'11. Berlin, Heidelberg: Springer-Verlag, 2011, pp. 171-184.

[6] H. Bagheri, M. Schmitt, and X. X. Zhu, "Uncertainty assessment and weight map generation for efficient fusion of TanDEM-X and Cartosat-1 DEMS," ISPRS - International Archiv of the Photogrammetry, Remote Sens. and Spatial Information Sci., vol. XLII-1/W1, pp. 433-439, 2017.

[7] M. Schmitt and X. X. Zhu, "Data Fusion and Remote Sensing: An ever-growing relationship," IEEE Geoscience and Remote Sens. Magazine, vol. 4, no. 4, pp. 6-23, dec 2016.

[8] L. I. Rudin, S. Osher, and E. Fatemi, "Nonlinear total variation based noise removal algorithms," Physica D: Nonlinear Phenomena, vol. 60, no. 1-4, pp. 259-268, nov 1992.

[9] G. Kuschk, P. d'Angelo, D. Gaudrie, P. Reinartz, and D. Cremers, "Spatially Regularized Fusion of Multiresolution Digital Surface Models," IEEE Trans. on Geosci. and Remote Sens., vol. 55, no. 3, pp. 14771488, 2017.

[10] T. Pock, L. Zebedin, and H. Bischof, TGV-Fusion. Berlin, Heidelberg: Springer Berlin Heidelberg, 2011, pp. 245-258.

[11] R. Perko and C. Zach, "Globally optimal robust DSM fusion," European Journal of Remote Sens., vol. 49, no. 1, pp. 489-511, 2016. 